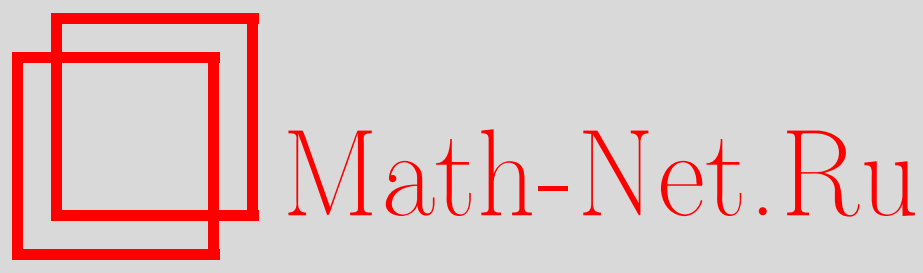

М. Пелозо, Можно ли примирить нарушенную вращательную инвариантность с инфляцией?, ТМФ, 2012, том 170, номер 2, 223-229

DOI: https://doi.org/10.4213/tmf6760

Использование Общероссийского математического портала Math-Net.Ru подразумевает, что вы прочитали и согласны с пользовательским соглашением http://www . mathnet.ru/rus/agreement

Параметры загрузки:

IP : 52.23 .180 .231

26 апреля 2023 г., 14:45:11

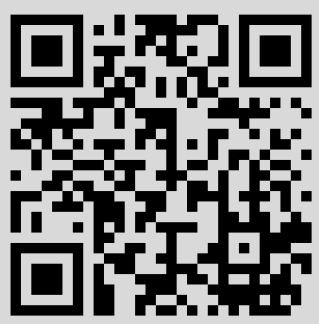




\title{
ФИЗИКА
}

Том 170, № 2

февраль, 2012

(C) 2012 г.

\section{М. Пелозо* \\ МОЖНО ЛИ ПРИМИРИТЬ НАРУШЕННУЮ ВРАЩАТЕЛЬНУЮ ИНВАРИАНТНОСТЬ С ИНФЛЯЦИЕЙ?}

\begin{abstract}
В литературе можно найти несколько численных моделей нарушенной вращательной инвариантности, реализующих этот эффект через векторное поле (поля) с ненулевым средним по пространственному вакууму, появление которых было вызвано утверждениями о нарушении вращательной инвариантности в данных космического зонда WMAP. Обсуждается, почему многие из этих моделей имеют ду́ховые неустойчивости.
\end{abstract}

Ключевые слова: нарушение вращательной инвариантности, духовая неустойчивость.

Посвящается памяти Льва Кофмана

Я хотел бы засвидетельствовать свою признательность Льву Кофману за то, что мне была предоставлена замечательная возможность работать с ним, и за то время, которое мы провели вместе. Наше сотрудничество оказало на меня огромное влияние, и мне повезло, что я знал человека и физика такого масштаба.

Сначала мой интерес к обсуждаемой в настоящей работе теме заключался в том, что я хотел понять, как ведут себя космологические возмущения при ослаблении предположения об изотропности и однородности фона. Это в свою очередь связано с вопросом о том, откуда мы на самом деле знаем, что наша Вселенная однородна и изотропна. Для определенности я изучил простейший случай фона Бьянки-I (по существу, имеется в виду вселенная с различными скоростями расширения в различных направлениях), который подвергался изотропизации в момент установления инфляции. Я заметил, что в анизотропном режиме одна из поляризаций гравитационной волны демонстирует значительный рост. Но я рассматривал это главным образом как любопытный с математической точки зрения факт. Лев, наоборот, сразу понял физическое происхождение этого эффекта и его возможные следствия. Источник этого роста коренится в неустойчивости пространств Казнера, которые исследовались в основополагающих работах [1]. Льва этот эффект привел в восхищение своей универсальностью: он описывает поведение широкого класса пространств, сжимающихся по направлению к сингулярности, причем эффект характеризуется переходом между различными геометриями Казнера (каждая из которых

${ }^{*}$ School of Physics and Astronomy, University of Minnesota, Minneapolis, MN, USA. E-mail: peloso@physics.umn.edu 
неустойчива; это так называемые колебания Белинского-Халатникова-Лифшица). Лев понял, что результат наших вычислений представляет собой то же самое явление для расширяющегося пространства Казнера, с тем единственным отличием, что теперь неустойчивость выключается, когда главным становится инфлатон и геометрия изотропизуется. В результате роста тензорной моды мог бы появиться сигнал от сильной гравитационной волны; это можно было бы наблюдать на самых больших масштабах при условии, что инфляция продолжается не слишком долго. Когда я ответил, что это не так привлекательно, как стандартные инфляционные предсказания, поскольку тем самым вводится зависимость от начальных условий, Лев возразил, что это напомнило ему доинфляционную эпоху, когда космологи были привычны к тому, что данные наших наблюдений должны зависеть от начальных условий, причем таким образом, который невозможно предсказать, опираясь на лежащую в основе теорию. Сейчас легко принять как само собой разумеющееся то "прикрытие", которое обеспечивает инфляция, отщепляя (возможно) существующие до начала наблюдений начальные условия от того, что нами наблюдается. Так это или нет - это, конечно, вопрос наблюдений, а не наших теоретических пристрастий. Таков был последний урок, который мне выпала честь получить от Льва.

Главная тема ряда исследований, которые обсуждаются в настоящей работе, это вопрос о том, возможно ли согласовать одну из так называемых аномалий данных космического зонда WMAP с инфляционной картиной и может ли это привести к новым предсказаниям. В настоящий момент зонд WMAP [2] дает самые лучшие данные по нашей Вселенной на самых больших масштабах, доступных для наблюдений. В течение ряда лет указывалось на присутствие аномалий в данных WMAP. Если эти аномалии имеют космологическое происхождение, то они требуют радикального переосмысления инфляционной картины.

Первое из таких утверждений касается очень больших масштабов (выравнивание между самыми большими кратными в разложении температурной анизотропии реликтового излучения [3]), для которых галактическое обрезание и удаление переднего плана могут составлять проблему. В более поздних работах обсуждались особенности в высших мультиполях (что соответствует меньшим масштабам). Например, в работе [4] было заявлено, что можно доказать существование нарушенной вращательной инвариантности путем рассмотрения мультиполей вплоть до $\ell=400$. А именно, утверждалось, что данные предполагают степенной первичный спектр с угловой зависимостью относительно "особого направления" на небе. Направление, указанное в этом исследовании, не имеет астрофизической значимости. Последующие работы [5] скорректировали этот анализ; появилось более значимое (порядка $9 \sigma)$ свидетельство аномалии. В настоящее время обнаружено, что особое направление почти совпадает с осью эклиптики, и это придает дополнительный вес его систематическому происхождению.

В многочисленных исследованиях аномалий данных WMAP предложены различные параметризации этих эффектов. Во многих случаях параметризация не основана на теоретической модели и, хотя она может служить полезной схемой для изучения данных, не улучшает наше теоретическое понимание аномалий. Некоторое улучшение достигнуто в ряде работ, в которых фактически предлагаются конкретные космологические модели для того, чтобы воспроизвести подобные особенности. В целом ряде моделей, вызывающих особый интерес, статистическая изотропия нарушена ненулевым пространственным вакуумным средним некоторого векторного 
поля (полей), что может привести или к небольшой анизотропии в инфляционном расширении [6]-[8], или к анизотропному механизму генерации первичных возмущений [9]. Все эти модели имеют некоторые неминимальные составляющие, которые противодействуют быстрой изотропизации (в данном случае - быстрому убыванию векторного вакуумного среднего), которая обычно имеет место во время инфляции. Даже если последние результаты работы [5] в сильной степени свидетельствуют против космологического происхождения этой аномалии, все же имеются различные мотивации для исследования таких моделей.

C феноменологической точки зрения спутник Planck [10], данные которого по реликтовому излучению будут опубликованы, как ожидается, летом 2012 г., предоставит нетривиальный способ проверить результаты зонда WMAP, поскольку если эффект нарушения вращательной инвариантности имеет космологическое происхождение, то он будет даже еще более значимым в данных спутника Planck (вследствие большей точности при больших $\ell$ ). С теоретической точки зрения, если рассматривать их реальную значимость, из моделей работ [6]-[9], по-видимому, следует, что на самом деле не так уж сложно нарушить статистическую изотропию, так что нам не следует принимать ее за сильный теоретический приоритет. В этом смысле сам термин "аномалия" представляется не вполне обоснованным: анизотропия могла бы просто быть дополнительным параметром, который легко получить из теории и который, как показывают наблюдения, должен быть малым или равным нулю.

Еще одна мотивировка нашей работы состоит в том, что в исходных предположениях статей [6]-[9] нет полного анализа космологических возмущений, и проведенные полностью исследования могли бы дать новые феноменологические предсказания, которые позволили бы подтвердить или опровергнуть эти модели как источник аномалии, указанный в работах [4], [5]. На неизотропном фоне скалярное и тензорное возмущения метрики взаимодействуют между собой уже на линеаризованном уровне. Это может привести к нестандартной (и, потенциально возможно, усиленной) амплитуде гравитационных волн и к значительной скалярно-тензорной корреляции [11] (поскольку эта величина обращается в нуль в стандартном случае, что было бы отличительным признаком анизотропии). Такие вычисления были на самом деле исходной целью наших работ, однако мы обнаружили, что модели из работ [6]-[9] имеют ду́ховые неустойчивости, которые, по нашему мнению, делают необоснованными всякие полученные для них феноменологические предсказания.

Явные и обстоятельные вычисления для моделей из работ [7] и [8], [9] можно найти в наших статьях [12] и [13] соответственно. Мы провели аналогичные вычисления для модели из статьи [6], но не опубликовали, поскольку их можно провести с помощью тех же методов. Из соображений краткости мы не будем здесь приводить эти расчеты. Вместо этого мы, пренебрегая возмущениями метрики, проделаем гораздо более простое вычисление, которое, однако, проясняет вопрос о том, почему появляются неустойчивости [14].

Начнем обсуждение с рассмотрения массивного вектора на однородном и изотропном фоне:

$$
S=\int d^{4} x \sqrt{-g}\left(-\frac{1}{4} F_{\mu \nu} F^{\mu \nu}-\frac{M^{2}}{2} A_{\mu} A^{\mu}\right) .
$$

Мы предполагаем, что $A_{\mu}$ имеет пренебрежимо малое вакуумное среднее, и разлагаем его флуктуации как $A_{\mu}=\left(\alpha_{0}, \partial_{i} \alpha_{L}+\alpha_{i}^{\mathrm{T}}\right)$. Поперечное векторное возмущение 
$\alpha_{i}^{\mathrm{T}}$, удовлетворяющее условию $\partial_{i} \alpha_{i}^{\mathrm{T}}=0$, содержит две физические моды. Эти моды ведут себя хорошо и отщепляются от возмущений $\alpha_{0}, \alpha_{\mathrm{L}}$. В дальнейшем мы ими пренебрегаем. При $M^{2} \neq 0$ два возмущения $\alpha_{0}, \alpha_{\mathrm{L}}$ кодируют одну дополнительную степень свободы - продольную векторную поляризацию. Действительно, мода $\alpha_{0}$ является нединамической, поскольку она появляется в действии без производных по времени, и ее следует устранить интегрированием. Уравнение движения для этой моды после разложения Фурье по пространственным направлениям дает $\alpha_{0}=\left[p^{2} /\left(p^{2}+M^{2}\right)\right] \dot{\alpha}_{\mathrm{L}}$, где $p=k / a-$ физический импульс моды, $k-$ сопутствующий импульс, а точка над символом обозначает дифференцирование по времени. Подставляя это решение в (1), получаем действие для динамической моды:

$$
S_{\mathrm{L}}=\int d t d^{3} k a^{3} \frac{p^{2} M^{2}}{2}\left(\frac{\left|\dot{\alpha}_{L}\right|^{2}}{p^{2}+M^{2}}-\left|\alpha_{\mathrm{L}}\right|^{2}\right) .
$$

Существование продольной векторной моды обусловлено массовым членом, поэтому не удивительно, что кинетический член умножен на $M^{2}$. Мы видим, что эта мода является духом в ультрафиолетовом пределе при $M^{2}<0$. Те же самые выводы следуют и из вычисления пропагатора векторного поля или из использования формализма Штюкельберга [12]. Подчеркнем аналогию со случаем массивной гравитации, где массовый член также контролирует устойчивость. А именно, дух появляется на линеаризованом уровне, за исключением случая, когда масса имеет в точности тип Фирца-Паули. Соображения устойчивости применимы, даже если продольная мода не является отдельным скалярным полем, а представляет собой просто одну из поляризаций массивного вектора или тензора.

Теперь покажем, что в моделях из работ [6], [8], [9] величина $M^{2}$ должна иметь неправильный знак (мы отсылаем читателя к работам [12], [14] за обсуждением модели из статьи [7]). Мы предполагаем для определенности, что пространственное вакуумное среднее вектора направлено вдоль оси $x$. Это дает элемент длины

$$
d s^{2}=-d t^{2}+a(t)^{2} d x^{2}+b(t)^{2}\left(d y^{2}+d z^{2}\right) .
$$

Введем две скорости расширения $H_{a} \equiv \dot{a} / a, H_{b} \equiv \dot{b} / b$ и определим их среднее $H$ и перемасштабированную разность $h$ как $H \equiv\left(H_{a}+2 H_{b}\right) / 3$ и $h \equiv\left(H_{b}-H_{a}\right) / 3$. Инфляционные расширения, которые мы рассматриваем ниже, характеризуются постоянными или медленно эволюционирующими скоростями. Для рассматриваемых моделей $h / H=O\left(B^{2}\right)$, где $B$ - перемасштабированное вакуумное среднее векторного поля: $\left\langle A_{x}\right\rangle \equiv M_{p} a B[6]$, [8], поэтому $B$ также должно медленно скатываться во время режима медленного скатывания. Рассмотрим феноменологически адекватный случай $B<1$ умеренной анизотропии. Чтобы добиться медленного скатывания, рассмотрим действие

$$
S=\int d^{4} x \sqrt{-g}\left(\frac{M_{p}^{2}}{2} R-\frac{F^{2}}{4}-V\left(A^{2}\right)+\frac{\xi}{2} R A^{2}\right),
$$

где вакуумная энергия, ведущая к космологическому расширению, включена в $V$. Разлагая потенциал вплоть до квадратичного порядка по $A_{\mu}$ и сравнивая с $(1)$, получаем из данного действия массовый член

$$
M^{2}=2 \frac{\partial V}{\partial A^{2}}-\xi R=2 \frac{\partial V}{\partial A^{2}}-6 \xi\left(2 H^{2}+h^{2}+\dot{H}\right) .
$$


Уравнение движения для перемасштабированного вакуумного среднего $B$, полученное из (2), имеет вид $\ddot{B}+3 H \dot{B}+\mathcal{Q} B=0$, где

$$
\mathcal{Q} \equiv 2 \frac{\partial V}{\partial A^{2}}-2 H h-5 h^{2}-2 \dot{h}+(1-6 \xi)\left(2 H^{2}+h^{2}+\dot{H}\right) .
$$

Условие медленного скатывания $B$ требует выполнения условия $\mathcal{Q} \ll H^{2}$ (поскольку член $3 H \dot{B}$ определяет "сопротивление" движению). В работе [6] изучались решения с постоянными $H_{a, b}$ в отсутствие члена $A^{2} R$, т. е. при $\xi=0$. Таким образом, требуется выполнение условия $\mathcal{Q}=0$ или, другими словами, неравенства

$$
\frac{\partial V}{\partial A^{2}}=-H^{2}+H h+2 h^{2}=-H_{a} H_{b}<0 .
$$

Это соответствует отрицательному квадрату массы в уравнении (3). В работе [8] получено медленное скатывание с достаточно малым значением $\partial V / \partial A^{2}$ и с $\xi=1 / 6$. Это также дает отрицательный квадрат массы в уравнении (3). Аналогично, модель из работы [9] характеризуется действием (2) с $\xi=1 / 6$ (данное требование в этом случае связано с масштабной инвариантностью получающихся возмущений кривизны), поэтому тоже имеет дух, даже если фоновое решение изотропно (на самом деле следует сказать “даже если фоновая анизотропия очень мала"; строго говоря, ненулевое векторное вакуумное среднее, которое должно иметь место и для модели из работы [9], приводит к небольшой анизотропии, поэтому и для этой модели актуальное вычисление устойчивости именно такое, о котором сообщается в разделах IV и $\mathrm{V}$ работы [13]).

Добавление возмущений метрики значительно усложняет вычисления, но не изменяет сформулированные выше заключения. Более точно, одно возмущение (которое принимает вид продольной моды в пределе, когда гравитация отщепляется) превращается вблизи пересечения горизонта из хорошо себя ведущей моды в дух. Когда это происходит, линеаризованные решения для возмущений (включая $\delta g_{\mu \nu}$ ) расходятся. Для модели из работы [8], в которой присутствует больше векторов, было найдено, что некоторые возмущения ведут себя именно таким образом, в то время как некоторые другие являются духами в течение всего режима под горизонтом. Кроме того, было найдено, что если добавить к модели из работы [9] малый положительный массовый член (такой массовый член уже присутствует в работе [8]), то возмущения также расходятся, когда полная масса $M^{2}$ обращается в нуль.

Представленные в настоящей работе вычисления справедливы на линеаризованном уровне. Можно утверждать, что полученная нами неустойчивость проявляется только на этом уровне, однако это до некоторой степени можно исправить с помощью нелинейных взаимодействий. В то время как данную возможность априори нельзя отбросить, следует иметь в виду, что все феноменологические предсказания, приводимые в литературе для указанных выше моделей, основаны на линеаризованных вычислениях. Доверять таким предсказаниям не следует, тем более, что они основаны на приближенном рассмотрении линеаризованой системы для возмущений, тогда как выполненные нами полные линеаризованные вычисления показывают, что решения на самом деле расходятся. Что касается нелинейных взаимодействий, можно ожидать, что они только усугубят проблему, поскольку взаимодействие между здоровым полем и духом приводит к распаду вакуума.

Таким образом, включающие духи теории можно рассматривать лишь как эффективные теории поля, которые верны только на масштабе, задаваемом массовым 
членом. В действительности все эти теории требуют обрезания, что делает их неприменимыми при высоких энергиях вне зависимости от знака массового члена. Это можно понять, если посмотреть на поведение массивных векторных полей при высоких энергиях. Исследуемые модели обладают калибровочной инвариантностью, которая жестко нарушается явным массовым членом $M^{2} A^{2}$ для вектора. Хорошо известно, что в этом случае взаимодействия продольных бозонов нарушают унитарность на масштабе, который параметрически задается величиной $M$, что приводит к выходу квантовой теории из-под контроля. Для предлагаемых моделей $M$ равна постоянной Хаббла или меньше нее, так что весь подгоризонтный режим может оказаться некорректно определенным. Хотя нам известно о явных вычислениях этой задачи только в пространстве-времени Минковского, мы считаем, что их также можно применить к инфляционному случаю, если имеются ненарушенная лоренцевская инвариантность и переходы к локально плоской системе отсчета (и, более того, во время инфляции массовый член для вектора почти постоянен, а импульс меняется адиабатически в подгоризонтном режиме). Поскольку эта проблема присутствует при обоих знаках $M^{2}$, "излечивающая" теория, в которой имеется жесткая векторная масса и дух, несет в себе больше проблем, чем излечивающая теория только с жесткой векторной массой.

Наиболее непосредственное доопределение теории с жесткой массой в ультрафиолетовом пределе - это доопределение через механизм Хиггса. Масса тогда должна быть обусловлена вакуумным средним скалярного поля, которое становится динамическим выше масштаба $M$. Таким образом, теория остается под контролем и в коротковолновом режиме, и можно применить все стандартные вычисления, справедливые для скалярных полей во время инфляции. Однако если $M^{2}$ должна иметь неправильный знак, то скалярное поле в этой ультрафиолетово полной теории должно быть духом. В действительности, когда утверждается, что теория с духами справедлива только как эффективная теория, предполагается, что свободная от духов ультрафиолетово полная теория существует. Однако нам не известно никаких явных конструкций, которые это реализуют.

Недавно именно для того чтобы избежать неустойчивостей, которые мы обсуждали, были предложены некоторые модели [15], [16]. Хотя эти модели и сложнее, они интересны, поскольку являются доказательствами существования того, что быстрой инфляционной изотропизации действительно можно избежать контролируемым образом. Они также составляют первые полные реализации, в которых анизотропные сигнатуры можно конкретно вычислить. Модель из статьи [15] характеризуется взаимодействием между кинетическим членом векторного поля и функцией инфляции $f(\phi) F^{2}$. При подходящем выборе $f$ модель может допускать медленно скатывающееся анизотропное инфляционное решение. Поскольку для вектора массовый член отсутствует, данная модель свободна от духовых неустойчивостей. В работе [17] впервые было показано, что характерная скалярно-тензорная корреляция много меньше того значения, которое следовало бы из величины анизотропии в фоновом расширении. В работе [16] модель, наоборот, характеризуется внешними зависящими от времени функциями, на которые умножены кинетический и массовый члены вектора. Для подходящей временно́й зависимости векторное поле дает масштабно-инвариантый и статистически изотропный степенной спектр. Было бы интересно обобщить данную модель, заменив эти внешние функции на функции динамического скалярного поля. 
В завершение сделаем несколько замечаний о цитируемой литературе. В настоящей работе я сфокусировал свое внимание только на работах, соавтором которых являюсь, а также на тех, которые существенно важны для повествования. По поводу более исчерпывающего обзора и, конечно, чтобы воздать должное действительно актуальным работам, я отсылаю заинтересованного читателя к списку литературы в двух наших последних статьях [13], [17] на данную тему.

Благодарности. Я благодарен организаторам XVI Международного семинара по физике высоких энергий "Quarks-2010" за очень стимулирующую конференцию. Настоящая статья основана на совместной работе с К. Контальди, А. Э. Гюмрюкоглу, Б. Химетоглу и Л. Кофманом; отмечу также очень полезные обсуждения с Н. Калопером. Моя работа частично поддержана со стороны US Department of Energy (грант DE-FG02-94ER-40823).

\section{Список литературы}

[1] E. M. Lifshitz, I. M. Khalatnikov, Adv. Phys., 12:46 (1963), 185-249; V.A. Belinsky, I. M. Khalatnikov, E. M. Lifshitz, Adv. Phys., 19:80 (1970), 525-573; 31:6 (1982), 639-667.

[2] Wilkinson Microwave Anisotropy Probe (WMAP), lambda.gsfc.nasa.gov.

[3] M. Tegmark, A. de Oliveira-Costa, A. Hamilton, Phys. Rev. D, 68:12 (2003), 123523, 14 pp., arXiv: astro-ph/0302496; K. Land, J. Magueijo, Phys. Rev. Lett., 95:7 (2005), 071301, 4 pp., arXiv: astro-ph/0502237.

[4] N. E. Groeneboom, H. K. Eriksen, Astrophys. J., 690:2 (2009), 1807-1819, arXiv: 0807.2242.

[5] D. Hanson, A. Lewis, Phys. Rev. D, 80:6 (2009), 063004, 15 pp., arXiv: 0908.0963; N.E. Groeneboom, L. Ackerman, I. K. Wehus, H. K. Eriksen, Bayesian analysis of an anisotropic universe model: systematics and polarization, arXiv: 0911.0150.

[6] L. H. Ford, Phys. Rev. D, 40:4 (1989), 967-972.

[7] L. Ackerman, S. M. Carroll, M. B. Wise, Phys. Rev. D, 75:8 (2007), 083502, 7 pp., arXiv: astro-ph/0701357; Erratum, 80:6 (2009), 069901.

[8] A. Golovnev, V. Mukhanov, V. Vanchurin, JCAP, 06 (2008), 009, 7 pp., arXiv: 0802.2068.

[9] K. Dimopoulos, M. Karciauskas, D. H. Lyth, Y. Rodriguez, JCAP, 05 (2009), 013, 35 pp., arXiv: 0809.1055.

[10] Planck Science Team Home, arXiv: www.sciops.esa.int/index.php?project=PLANCK.

[11] A.E. Gümrükçüoğlu, C. R. Contaldi, M. Peloso, JCAP, 11 (2007), 005, 35 pp., arXiv: 0707.4179; A. E. Gümrükçüoğlu, L. Kofman, M. Peloso, Phys. Rev. D, 78:10 (2008), 103525, 21 pp., arXiv: 0807.1335.

[12] B. Himmetoglu, C. R. Contaldi, M. Peloso, Phys. Rev. D, 79:6 (2009), 063517, 19 pp., arXiv: 0812.1231.

[13] B. Himmetoglu, C. R. Contaldi, M. Peloso, Phys. Rev. D, 80:12 (2009), 123530, 36 pp., arXiv: 0909.3524.

[14] B. Himmetoglu, C. R. Contaldi, M. Peloso, Phys. Rev. Lett., 102:11 (2009), 111301, 4 pp., arXiv: 0809.2779.

[15] M. Watanabe, S. Kanno, J. Soda, Phys. Rev. Lett., 102:19 (2009), 191302, 4 pp., arXiv: 0902.2833.

[16] K. Dimopoulos, M. Karciauskas, J. M. Wagstaff, Phys. Lett. B, 683:4-5 (2010), 298-301, arXiv: 0909.0475.

[17] A. E. Gümrükçüoğlu, B. Himmetoglu, M. Peloso, Phys. Rev. D, 81:6 (2010), 063528, 16 pp., arXiv: 1001.4088. 